\title{
Impairments in balance and mobility identify delirium in patients with comorbid dementia
}

Corresponding author: Neus Gual. Parc Sanitari Pere Virgili, Avinguda Vallcarca 169205, 08023 Barcelona, Spain. ngual@perevirgili.cat (+34) 932594000

Dr Sarah J Richardson, Institute of Neuroscience, Biomedical Research Building, Campus for Ageing and Vitality, Newcastle University, Newcastle upon Tyne, UK.

Dr Daniel H J Davis, MRC Unit for Lifelong Health and Ageing at UCL, London, UK Dr Giuseppe Bellelli, School of Medicine and Surgery, University of Milano-Bicocca, Milan, Italy

Dr Wolfgang Hasemann, Universitatsspital Basel, Basel, Switzerland

Professor David Meagher, Graduate Entry Medical School, University of Limerick, Limerick, Ireland

Dr Stefan H Kreisel, Evangelisches Krankenhaus Bielefeld, Department of Psychiatry and Psychotherapy Bethel, Division of Geriatric Psychiatry, Bielefeld, Germany

Professor Alasdair M J MacLullich, Edinburgh Delirium Research Group, Geriatric Medicine, University of Edinburgh, Edinburgh, UK

Dr Joaquim Cerejeira, Department of Psychiatry, Hospitais da Universidade de Coimbra, Coimbra, Portugal

Dr Marco Inzitari, Parc Sanitari Pere Virgili, Barcelona, Spain.

Dr Alessandro Morandi, Ancelle Hospital, Cremona, Italy. 


\section{INTRODUCTION}

Delirium Superimposed on Dementia (DSD), has a prevalence ranging from 22 to $89 \%$ (Fick et al, 2002; Morandi et al., 2016) and is associated with worse outcomes than each condition separately, including functional decline, institutionalization, mortality, and greater health care costs compared with patients with dementia alone (Bellelli et al., 2007; Fick et al., 2013; Morandi et al., 2014; Dani et al., 2017; Richardson et al., 2017). Although cognitive impairment is one of the most important risk factors for developing delirium, the lack of a reference standard for DSD (Morandi et al., 2016) makes diagnosing delirium in patients with dementia a challenge. Indeed, because assessments of older people generally use cognitive tests to identify delirium, scores are usually confounded in patients with dementia regardless of whether they have delirium.

An alternative approach is to consider non-cognitive features of delirium and dementia. Previous work regarding motor function and delirium has shown that patients with delirium have a pattern of fluctuating motor performance related to the evolution of delirium. This pattern is appreciable in individuals with dementia developing delirium but not in patients with dementia alone (Bellelli et al., 2011). Therefore, motor performance may discriminate DSD from dementia. Though few studies have evaluated the association between delirium and motor fluctuations (Bellelli et al., 2011) it has been demonstrated that irrespective of dementia status, the presence of delirium is almost invariably characterized by discernible alterations to motor activity (Meagher et al., 2012).

The Hierarchical Assessment of Balance And Mobility (HABAM) is a valid and reliable tool designed for a rapid assessment of mobility and balance in hospitalized older people (MacKnight and Rockwood, 1995). As a global test of motor performance, it grades function from full, independent ambulation to complete inability to move. Nonetheless, its 
associations with delirium and dementia have not been comprehensively evaluated. This study aimed to explore whether assessing motor function using the HABAM may act as an indicator of delirium, and evaluate the utility of the HABAM in recognizing DSD in older people at admission to acute or rehabilitation settings.

\section{METHODS}

This was a cross-sectional analysis of consecutive patients aged $\geq 70$ years admitted to acute or rehabilitation settings in five Hospitals in Italy (Cremona, Monza), Ireland (Limerick), Portugal (Coimbra) and Switzerland (Basel). Exclusion criteria were the presence of aphasia, history of major stroke, coma at admission and poor vision or hearing. Informed consent was obtained from all participants (or by suitable proxies if delirium or cognitive impairment). The study protocol was approved by the local ethics committee at each center. The current sample has also been previously described (Richardson et al., 2017).

Patients were assessed for delirium and dementia at admission (within the first 72 hours) by a group of experienced and trained delirium clinician-researchers competent to diagnose delirium using DMS-5 and the delirium motor subtypes using the DMSS; as well as cognitive scales like s-MMSE and IQCODE. As per the HABAM manual, a pre-study training was carried out for those professionals to achieve the maximum compliance with the use of the instrument. The study protocol was established at regular meetings of the European Delirium Association but no formal tests of inter-rater reliability were conducted. After the initial evaluation, patients were then divided in 4 groups: no delirium and no dementia (normal comparison group 1); no delirium and dementia (group 2); delirium and no dementia (group 3); delirium and dementia (group 4). Delirium was diagnosed according to the DSM-5 criteria by using a standardized procedure, as previously described (Richardson et al., 2017). Pre-existing dementia in non-delirious patients was 
establish with scores $<28$ in standardized Mini-Mental State Examination (s-MMSE) (Molloy and Standish, 1997) and a score $\geq 3.5$ in the Questionnaire of Cognitive Decline in the Elderly (IQCODE). In delirious patients, pre-existing dementia was diagnosed according to an IQCODE score $\geq 3.5$ (Jorm et al., 1991). Formal tools for assessing altered arousal included the m-RASS and the Observational Scale of Level of Arousal (OSLA) and low scores were taken to indicate severe inattention. To classify the different motor subtype of delirium, the Delirium Motor Subtype Scale (DMSS) identified the patients in 4 groups: hyperactive, hypoactive, mixed and no motor group. Comorbidity was measured using the Charlson comorbidity index [higher score= greater comorbidity].

Mobility and balance at admission was assessed using the HABAM (MacKnight and Rockwood, 1995) at the same time as delirium status was determined. The HABAM evaluates mobility (from needs positioning in bed to unlimited mobility), transfers (from total lift to independent and vigorous) and ambulation (from impaired statistic sitting to stable ambulation). It is designed to be simple and quick to use, and avoids the floor effect of many other tools in those unable to stand, scoring a total of 27 points maximum as a sum of scores from the three domains (MacKnight and Rockwood, 1995). Higher scores are indicative of greater mobility impairment. HABAM has a good intra-rater and inter-rater reliability for individual monitoring ( $r=0.92$ and 0.91 , respectively), with adequate concurrent validity as a measure of function in balance and mobility (Rockwood et al., 2008). The HABAM is a continuous scale without defined cut-off points in terms of normal or abnormal performance, therefore we used the maximal Youden's index (sensitivity + specificity -1 ) to determine optimum test accuracy post hoc.

\section{Statistical Analysis.}

A univariate analysis was performed to analyze the differences in characteristics, of defined cut-off points, of patients with dementia, delirium, neither or both (chi-square test for dichotomous variables; ANOVA for continuous variable). To assess if HABAM scores 
were influenced by factors other than the presence of delirium, we performed a logistic multinomial regression, where the outcomes were the four diagnostic categories adjusted by potential confounders. Differences in motor subtypes in patients with and without dementia who developed delirium were assessed using the Fisher's exact test. Diagnostic test accuracy was assessed twice, for the whole sample and also stratified by dementia status, using receiver operating characteristic $(\mathrm{ROC})$ curves to determine the sensitivity, specificity, positive and negative likelihood ratios and also the area under the curve $(A \cup C)$, with confidence intervals of $95 \%$. Statistical analyses were performed using SPSS software version 19.0 and Stata version 13.1.

\section{RESULTS}

A total of 114 people were included (Basel, $n=15$; Coimbra, $n=26$; Cremona, $n=21$; Limerick, $n=28$; Monza $n=24)$. Mean age+SD was $82 \pm 7$ years; $54 \%(n=62)$ female, mean Charlson comorbidity index $=2.8 \pm 2 ; 43 \%$ admitted into rehabilitation settings. Dementia alone was present in $24.6 \%(n=28)$, delirium alone in $18.4 \%(n=21)$, DSD in $27.2 \%$ $(n=31)$ and $29.8 \%(n=34)$ did not have delirium or dementia (Table 1). Age, sex, comorbidity and hospital setting were similar in the 4 groups, without significant differences in the univariate analysis.

The subtype frequency for patients with delirium ( $n=52 ; 45.6 \%)$ was: hyperactive subtype $(n=21)$, hypoactive $(n=20)$, mixed $(n=6)$ and no motor subtype $(n=5)$. Using Fisher's exact test, there was no significant difference in the motor subtype distribution of delirious patients with and without dementia $(p=0.30)$.

We observed higher HABAM scores in the groups with delirium. Using ANOVA performed on the whole sample (patients with and without pre-existing dementia), we found significantly higher HABAM scores in patients with delirium as compared to those without 
delirium (mean score $+S D=18.5+8.8$ vs $11.1+8.7 ; F=7.6 ; \mathrm{df}=1,105 ; p<0.001$ ). Moreover, among patients with preexisting dementia, those with delirium had a mean HABAM score 7 points higher than those without delirium ( $19.8 \pm 8.7$ vs $12.5 \pm 9.5 ; F=4.1$; $d f=1,53$; $\mathrm{p}<0.001$ ) (Table 1). In multinomial logistic regression adjusted by age, sex, setting and comorbidity, DSD, delirium alone and dementia alone were all associated with higher HABAM scores ((OR 1.2 [1.1 to 1.3], Wald chi-square 17.1, df=1, $\mathrm{p}<0.001) ;(\mathrm{OR} 1.1$ [1.1 to 1.2 ], Wald 4.9, $\mathrm{df}=1, \mathrm{p}=0.027)$; (OR 1.08 [1.01 to 1.16$]$, Wald $10.2, \mathrm{df}=1, \mathrm{p}=0.001$ ) respectively).

In the ROC curves, assessing the whole sample, a HABAM cut-off at 18 correctly classified $69 \%$ (sensitivity $67 \%$, specificity $71 \%$, AUC 0.74 ) of patients with delirium, compared with those without delirium. Assessing only patients with dementia, a HABAM cutoff at 22 correctly classified $70 \%$ (sensitivity $61 \%$, specificity $79 \%$, AUC 0.76 ) of patients with delirium superimposed on dementia from those with dementia alone (Figure 1)

\section{DISCUSSION}

In this study, patients with delirium had a higher score in the HABAM test, indicating more impaired motor function, compared with patients without delirium. HABAM scores were significantly higher in patients with pre-existing dementia and delirium. The overall accuracy of the HABAM test for differentiating DSD from dementia alone was good (AUC 0.76). Taken together, these findings emphasize the relative utility of considering motor aspects of delirium in order to identify DSD.

The diagnosis of DSD is a key challenge, and there is increasing interest in using noncognitive assessments to separate the diagnoses. This includes tasks assessing level of arousal and the ability to engage the patient in cognitive assessment including attention tests (Richardson et al., 2017). The m-RASS, a test of arousal, has also been investigated in patients with pre-existing dementia, demonstrating moderate sensitivity and 
very high specificity for the detection of DSD (Chester et al., 2012; Morandi et al., 2016). Our current findings build on other characterizations of delirium and motor dysfunction in DSD using the Tinetti scale and the Truck Control Test (Bellelli et al., 2011). While it appears the HABAM scale can identify important features of delirium in DSD cross-sectionally, serial fluctuations in motor function requires further study, especially after delirium resolution. Comparing to other assessments previously reported from this same study, the HABAM did not perform as well as other assessments, for example combining OSLA and attention tasks (with sensitivity 94\%, specificity 92\%, AUROC 0.98 in patients with previous dementia) (Richardson et al., 2017). While the diagnostic test accuracy for HABAM is modest overall, the prospect of an entirely motor, non-cognitive assessment in detecting older people with delirium may have direct clinical utility in settings where cognitive tests are infrequently performed.

This study has strengths along with limitations. First this was a multicenter study including data from different countries, and across different clinical settings. Second the assessment of delirium was conducted with a standardized procedure. However, the crosssectional nature of the study is limited by the focus upon prevalent delirium at the time of assessment. Therefore, we did not assess fluctuations in motor function during the delirium episode. Another limitation was that the analysis was not adjusted for patient's medication, which could be a potential confounder when analyzing motor function. One last limitation is that the cut-offs were chosen according to their overall accuracy in delirium identification but in real world practice sensitivity often assumes a greater importance such that use of the HABAM as a screening tests may require emphasizing sensitivity over specificity in order to minimize the frequency of false negative cases.

\section{CONCLUSIONS}


This study emphasizes the association between delirium and motor function and shows that people with delirium have identifiable impairments of worse motor function than those without delirium, regardless of presence of underlying dementia or clinical subtype. As a consequence, assessment of motor function in older people at hospital admission and during the hospital stay might help in the detection of DSD and the HABAM may provide a suitable brief tool for this purpose. Our understanding of the concept of DSD, particularly regarding motor fluctuations, is evolving and can be informed by further studies of disturbances to motor function. Serial assessment studies in particular can explore the extent to which changes in motor function indicate emerging delirium and may be especially useful in patients with pre-existing cognitive impairments such as dementia.

\section{CONFLICT OF INTEREST}

None

\section{DESCRIPTION OF AUTHORS' ROLES}

Study conception and design was done by all the authors. Acquisition of data was done by G. Bellelli, D. Meagher, A. Morandi, W Hasemann and J. Cerejeira. Data analysis was done by A. Morandi, D. Davis, S. Richardson and N. Gual. Interpretation of results was done by all the authors. Drafting, critical revision and final approval of the manuscript was done by all the authors. 


\section{Bibliography}

Bellelli, G., Frisoni, G. B., Turco, R., Lucchi, E., Magnifico, F., and Trabucchi M. (2007) 'Delirium superimposed on dementia predicts 12-month survival in elderly patients discharged from a postacute rehabilitation facility.', The journals of gerontology. Series A, Biological sciences and medical sciences, 62(11), pp. 1306-9.

Bellelli, G., Speciale, S., Morghen, S., Torpilliesi, T., Turco, R. and Trabucchi, M. (2011) 'Are fluctuations in motor performance a diagnostic sign of delirium?', Journal of the American Medical Directors Association, 12(8), pp. 578-83. doi: 10.1016/j.jamda.2010.04.010.

Chester, J. G., Beth Harrington, M., Rudolph, J. L. and VA Delirium Working Group (2012) 'Serial administration of a modified Richmond Agitation and Sedation Scale for delirium screening.', Journal of hospital medicine, 7(5), pp. 450-3. doi: 10.1002/jhm.1003.

Dani, M. Owen, L. H., Jackson, T. A., Rockwood, K. , Sampson, E. L. and Davis D. (2017) 'Delirium, frailty and mortality: interactions in a prospective study of hospitalized older people.', The journals of gerontology. Series A, Biological sciences and medical sciences. doi: 10.1093/gerona/glx214.

Fick, D. M., Steis, M. R., Waller, J. L. and Inouye, S. K. (2013) 'Delirium superimposed on dementia is associated with prolonged length of stay and poor outcomes in hospitalized older adults.', Journal of hospital medicine, 8(9), pp. 500-5. doi: 10.1002/jhm.2077.

Fick, D. M., Agostini, J. V and Inouye, S. K. (2002) 'Delirium superimposed on dementia: a systematic review.', Journal of the American Geriatrics Society, 50(10), pp. 1723-32.

Jorm, A. F., Scott, R., Cullen, J. S., and MacKinnon A. J. (1991) 'Performance of the Informant Questionnaire on Cognitive Decline in the Elderly (IQCODE) as a screening test for dementia.', Psychological medicine, 21(3), pp. 785-90.

MacKnight, C. and Rockwood, K. (1995) 'A Hierarchical Assessment of Balance and Mobility.', Age and ageing, 24(2), pp. 126-30.

Meagher, D. J., Leonard, M., Donnelly, S., Conroy, M., Adamis, D. and Trzepacz, P.T. (2012) 'A longitudinal study of motor subtypes in delirium: Frequency and stability during episodes', Journal of Psychosomatic Research, 72(3), pp. 236-241. doi: 10.1016/j.jpsychores.2011.11.013.

Meagher, D. J. et al. (2008) 'Motor symptoms in 100 patients with delirium versus control subjects: comparison of subtyping methods.', Psychosomatics, 49(4), pp. 3008. doi: 10.1176/appi.psy.49.4.300.

Molloy, D. W. and Standish, T. I. (1997) 'A guide to the standardized Mini-Mental State Examination.', International psychogeriatrics, 9 Suppl 1, pp. 87-94-50.

Morandi, A. et al. (2014) 'Delirium superimposed on dementia strongly predicts worse outcomes in older rehabilitation inpatients.', Journal of the American Medical Directors Association, 15(5), pp. 349-54. doi: 10.1016/j.jamda.2013.12.084. 
Morandi, A. et al. (2016) 'Detecting Delirium Superimposed on Dementia: Evaluation of the Diagnostic Performance of the Richmond Agitation and Sedation Scale.', Journal of the American Medical Directors Association, 17(9), pp. 828-33. doi: 10.1016/j.jamda.2016.05.010.

Morandi, A. et al. (2016) 'The Diagnosis of Delirium Superimposed on Dementia: An Emerging Challenge.', Journal of the American Medical Directors Association. doi: 10.1016/j.jamda.2016.07.014.

Richardson, S. J. et al. (2017) 'Detecting delirium superimposed on dementia: diagnostic accuracy of a simple combined arousal and attention testing procedure.', International psychogeriatrics, 29(10), pp. 1585-1593. doi: 10.1017/S1041610217000916.

Rockwood, K., Andrew MK., Mitnitski A. (2008) 'Reliability of the hierarchical assessment of balance and mobility in frail older adults.', Journal of the American Geriatric Society, 57(7), pp. 1213-1217. doi: 10.1111/j.1532-5415.2008.01773 
Table 1: Patients characteristics according to the presence of delirium and/or dementia.

\begin{tabular}{|c|c|c|c|c|c|}
\hline & $\begin{array}{c}\text { No delirium } \\
\text { No demen- } \\
\text { tia } \\
\mathrm{N}=34\end{array}$ & $\begin{array}{l}\text { Dementia } \\
\text { without de- } \\
\text { lirium } \\
\mathrm{N}=28\end{array}$ & $\begin{array}{c}\text { Delirium } \\
\text { without De- } \\
\text { mentia } \\
\mathrm{N}=21\end{array}$ & $\begin{array}{c}\text { Delirium } \\
\text { and De- } \\
\text { mentia } \\
\mathrm{N}=31\end{array}$ & $p^{4,5}$ \\
\hline Age & $80.7 \pm 6.1$ & $81.7 \pm 6.6$ & $84.2 \pm 6.3$ & $83.2 \pm 6.8$ & 0.344 \\
\hline Female & $19(55.8)$ & $14(50)$ & $11(52.4)$ & $18(58.1)$ & 0.931 \\
\hline Charlson Index & $2.4 \pm 2.5$ & $2.8 \pm 1.7$ & $2.8 \pm 1.5$ & $3.3 \pm 1.9$ & 0.336 \\
\hline $\begin{array}{l}\text { Rehabilitation set- } \\
\text { tings }\end{array}$ & 14 (41.2) & $14(50)$ & 7 (33.3) & $14(45.1)$ & 0.879 \\
\hline s-MMSE ${ }^{1}$ & $26.8 \pm 2.9$ & $16.1 \pm 5.8$ & - & - & $<0.001$ \\
\hline IQCODE $^{2}$ & $2.7 \pm 1.1$ & $4.2 \pm 0.6$ & $3.5 \pm 0.9$ & $4.4 \pm 0.6$ & $<0.001$ \\
\hline $\mathrm{HABAM}^{3}$ total & $9.9 \pm 8.0$ & $12.5 \pm 9.5$ & $16.6 \pm 8.9$ & $19.8 \pm 8.7$ & $<0.001$ \\
\hline
\end{tabular}

Chi-square test for dichotomous variables; ANOVA for continuous variables.

Values are expressed as mean \pm SD or $\mathrm{N}(\%)$.

1 Standardized Mini-Mental State Examination

${ }^{2}$ Questionnaire of Cognitive Decline in the Elderly

${ }^{3}$ HABAM: Hierarchical Assessment of Balance And Mobility.

${ }^{4} \mathrm{df}$ for all categories $=3$

${ }^{5}$ Numerater df for the F-tests is 1 and denominator df: 103 for Age, IQCODE and HABAM; 80 for Charlson Index, 97 for s-MMSE 